\title{
Diagnóstico y solución a la injusticia en Nancy Fraser e Iris Young. ¿Redistribución o reconocimiento?
}

David Alejandro Valencia Gutiérrez

Corporación Universitaria Minuto de Dios,

Colombia 


\title{
Diagnóstico y solución a la injusticia en Nancy Fraser e Iris Young. ¿Redistribución o reconocimiento?*
}

\begin{abstract}
Resumen: en la década de los noventa se presentó un debate académico entre Nancy Fraser e Iris Young. Estas autoras discutían sobre la forma como debería entenderse la justicia social para que fuera emancipadora. Por un lado, Young sugería que la injusticia debía definirse como opresión entendida como limitación de la participación política y la justicia por oposición como la ausencia de opresión. Por otro lado, Fraser sugería que la injusticia debía abordarse desde una perspectiva bidimensional de redistribución y reconocimiento para permitir la 'paridad participativa'. Este artículo aborda este debate para tomar postura a favor de Fraser debido a que su modelo es más inclusivo y logra responder satisfactoriamente a los problemas que Young plantea respecto a la opresión, pero también las cuestiones redistributivas que Young obvia.
\end{abstract}

Palabras clave: redistribución, reconocimiento, opresión, paridad participativa, justicia social.

\section{Diagnosis and solution to injustice in Nancy Fraser and Iris Young. Redistribution or recognition?}

\begin{abstract}
In the nineties an academic debate was presented between Nancy Fraser and Iris Young. These authors discussed the way in which social justice should be understood in order to be emancipatory. On the one hand, Young suggested that injustice should be defined as oppression understood as a limitation of political participation and oppositional justice as the absence of oppression. On the other hand, Fraser suggested that injustice should be approached from a two-dimensional perspective of redistribution and recognition to allow for 'participatory parity'. This article approaches this debate to take a position in favor of Fraser because his model is more inclusive and manages to respond satisfactorily to the problems that Young poses with respect to oppression, but also the redistributive issues that Young ignores.
\end{abstract}

Keywords: redistribution, recognition, oppression, participatory parity, social justice.

Fecha de recepción: 25 de marzo de 2018

Fecha de aceptación: 19 de noviembre de 2018

Forma de citar (APA): Valencia-Gutiérrez, D. (2019). Diagnóstico y solución a la injusticia en Nancy Fraser e Iris Young. ¿Redistribución o reconocimiento? Revista Filosofía UIS, 18(1), doi: http://dx.doi.org/10.18273/revfil.v18n1-2019003

Forma de citar (Harvard): Valencia-Gutiérrez, D. (2019). Diagnóstico y solución a la injusticia en Nancy Frase e Iris Young. ¿Redistribución o reconocimiento? Revista Filosofía UIS, 18(1),67-88.

David Alejandro Valencia Gutiérrez: colombiano. Magíster en Filosofía. Docente líder de investigación, Corporación Universitaria Minuto de Dios.

Correo electrónico: david.valencia@uniminuto.edu.co; david.filosofiaquial@gmail.com

ORCID: https://orcid.org/0000-0002-8277-5306

"Artículo de reflexión derivado de investigación. 


\section{Diagnóstico y solución a la injusticia en Nancy Fraser e Iris Young. ¿Redistribución o reconocimiento?}

\section{Introducción}

Este artículo pretende demostrar que las teorizaciones de Fraser en el nivel filosófico normativo, fundadas en el principio de igual valor moral, son más deseables que la propuesta de Young, que critica el ideal de imparcialidad y sugiere una sociedad donde se afirmen todas las diferencias.

Por lo anterior, se va a analizar el debate que Fraser y Young sostienen a lo largo de la década de los noventa y la influencia que Young ejerce en la obra posterior de Fraser. El debate inicia con el libro de Young La justicia y la política de la diferencia, publicado en 1990, al que Fraser objeta con dos textos: "Cultura, economía política y diferencia. Sobre el libro de Iris Marion Young: Justicia y la política de la diferencia" y "¿De la redistribución al reconocimiento? Dilemas en torno a la justicia en una época 'post-socialista'". La distancia entre las dos autoras se profundiza en el cruce de textos que fueron recogidos en el libro Theorizing Multiculturalism (1998), editado por Cynthia Willett: donde Young publica, "Unruly Categories: A critique of Nancy Fraser's dual systems theory" y Fraser, "A rejoinder to Iris Marion Young"1.

Para desarrollar el objetivo expuesto procederé en tres pasos: en primer lugar, expondré los planteamientos centrales de las dos autoras para ubicar las principales diferencias entre sus teorías. En segundo lugar, presentaré el debate directo que sostienen para contrastar sus posturas e identificar fortalezas y debilidades en cada autora. Finalmente, mostraré los motivos por los que considero que Fraser tiene una teoría más prometedora.

\section{Iris Young: Justicia, opresión y dominación}

Iris Young es una pensadora que articuló una crítica al paradigma distributivo y al ideal de imparcialidad liberal fundamento de su pensamiento. Estas líneas generales

\footnotetext{
1 A partir de aquí se usará la traducción portuguesa de estos dos textos: "Unruly Categories: A critique of Nancy Fraser's dual systems theory" y "A rejoinder to Iris Marion Young".
} 
se presentan en su artículo del año 1986 "The ideal of impartiality and the civic public" ${ }^{2}$, donde Young analiza las implicaciones del feminismo en la teoría moral y política. Su foco de análisis es la debilidad del ideal de imparcialidad liberal para definir criterios de justicia. Su tesis fundamental es que "el ideal de imparcialidad en la teoría moral expresa una lógica de la identidad que busca reducir las diferencias a una unidad" (Young, 2000, p. 167). Por lo tanto, en una sociedad heterogénea insistir en la imparcialidad es tratar de homogenizar a los grupos y de esta forma agredir los grupos sociales menospreciando sus rasgos distintivos.

Probablemente el aporte más destacado de Young es su crítica al paradigma distributivo de la justicia. Según Young, el paradigma distributivo tiene dos problemas. El primero es que "tiende a centrar el análisis en torno a la justicia social en la asignación de bienes materiales" (Young, 2000, p. 32). El segundo, es que "este enfoque tiende a ignorar la estructura social y el contexto institucional que a menudo contribuyen a determinar los modelos de distribución" (32). El paradigma distributivo en ocasiones intenta repartir bienes intangibles como el poder o el honor y con esto sugiere que éstos pueden ser tratados como cosas y así, ignora que no son cosas sino "funciones de relaciones y procesos sociales" (32). Con este panorama, el paradigma distributivo supone un modelo estático de la sociedad donde lo único que está por definir es la forma en la que las cosas deben ser repartidas dejando por fuera de la discusión política otros temas fundamentales que no tienen origen económico. Para superar el paradigma distributivo, Young considera que se deben abordar tres cuestiones que no son distributivas y que requieren de análisis en clave de justicia: los actuales procedimientos de toma de decisiones, la división del trabajo y, finalmente, los distintos símbolos y significados culturales.

La cultura es el elemento no distributivo más importante de todos. Su importancia radica en que "los significados simbólicos que la gente asocia con otro tipo de gente y con acciones, gestos o instituciones, a menudo afectan de modo significativo la situación social de las personas y sus oportunidades" (Young, 2000, p. 45). Al afectar la situación social de las personas es necesario que sea tematizada en un concepto amplio de justicia social. Entonces, lo que Young sugiere con estos elementos no distributivos es un 'desplazamiento del paradigma distributivo' que permita una ampliación del concepto de justicia social donde primen elementos no distributivos, especialmente los culturales, que ayuden a la vez a resolver los distributivos. Por lo anterior, sugiere Young que se reemplace el entendimiento económico de la injusticia para dar paso a las categorías de 'opresión' y 'dominación'.

El significado que le da Young al concepto de opresión es una acepción del término derivado de los movimientos estadounidenses de los 60 y no del uso

\footnotetext{
${ }^{2}$ La versión española de este artículo se encuentra publicado como el capítulo cuatro del libro Justicia y política de la diferencia (Young, 2000).
} 
clásico del término que se relaciona con la tiranía. "En su nuevo uso, la opresión designa las desventajas e injusticias que sufre alguna gente [...] por las prácticas cotidianas de una bien intencionada sociedad liberal" (Young, 2000, p. 74). Por lo tanto, Young condensa en su obra una definición de opresión propia de las víctimas y la pone al centro de su concepto de justicia social. Por su lado, la 'dominación' "consiste en la presencia de condiciones institucionales que impiden a la gente participar de la determinación de sus acciones" (68). De acuerdo a esta definición, se debe entender que la dominación se asocia con posturas institucionales que implícita o explícitamente limitan la participación de ciertos grupos sociales debido a sus características. También, 'dominación' en este sentido, implica una falla en los procesos democráticos que limitan la posibilidad de las personas y los grupos sociales a tomar decisiones que las afectan.

En consonancia con la opresión y la dominación, sugiere Young que el sujeto de la justicia sean los grupos sociales. Estos son definidos por la autora como "un colectivo de personas que se diferencian de al menos otro grupo a través de formas culturales, practicas o modos de vida" (Young, 2000, p. 77). Pero, "no todos los grupos sufren opresión en la misma medida" (Palacio, 2011, p. 84). Entonces, el análisis de Young arroja que la 'opresión' puede presentarse con cinco caras: explotación, marginación, carencia de poder, imperialismo cultural y la violencia ${ }^{3}$ (Pérez, 2010, p. 381).

\footnotetext{
La 'explotación' (1) es una categoría aparentemente distributiva, pero en la perspectiva de Young asume el papel de una relación de poder entre grupos. Young (2000) considera que, en la cara de la explotación, la opresión se presenta cuando los resultados del trabajo de un grupo se trasladan a otro de forma sostenida, haciendo que los esfuerzos de los grupos más desprotegidos terminen incrementando las riquezas y el poder de aquellos que tienen más.

De la marginación (2), sugiere Young, se desprenden dos tipos de injusticia no distributiva, que se suman a la carencia de bienes que son mitigados a través de programas de redistribución. La primera injusticia no distributiva es que las personas que reciben los subsidios quedan limitadas en sus derechos frente a quienes no dependen de dichos subsidios. La segunda, consiste en el bloqueo que los marginados experimentan para ejercer sus capacidades en los roles definidos por la sociedad (Cfr. Young, 2000, p. 95).

La tercera cara 'carencia de poder' (3) se presentan en la división del trabajo de los profesionales y los trabajadores no profesionales; si bien ambos terminan siendo presa de la explotación, los profesionales se encuentran en una condición privilegiada mientras que los no profesionales carecen de la "autoridad, estatus y sentido de sí misma que suelen tener los profesionales" (Young, 2000, p. 100).

El 'imperialismo cultural' (4) se presenta cuando "Las experiencias e interpretaciones de la vida social propias de los grupos oprimidos cuentan con pocas expresiones que afecten a la cultura dominante, mientras que esa misma cultura impone a los grupos oprimidos su experiencia e interpretación de la vida" (Young, 2000, p. 105). Es decir, la cultura dominante normaliza su modo de vida y sus significados culturales relegando a las otras culturas coexistentes a un estado de otredad e invisibilidad. Por un lado, la cultura dominante normalizada hace que los otros modos de vida no sean visibles y por el otro, excluye a los otros de la participación en los escenarios de toma decisión determinantes en la teoría de Young.

Por último, la quinta cara de la opresión, es la violencia (5) que se presenta cuando la humillación, el acoso e incluso las agresiones físicas se presentan de forma sistemática y normalizada contra los grupos sociales en desventaja. Según Young, y los índices de violencia contra la mujer, el hecho de ser mujer se convierte en una predisposición para ser víctima de una violación.
} 
Pero ante este panorama ¿cómo alcanzar la justicia? Respondería Young que superar la injusticia que sufren los grupos sociales en el marco de las caras de la opresión, requiere una superación del ideal de asimilación o 'principio de igualdad' que exige la neutralidad del Estado frente a la diferencia. Este ideal es negativo para Young porque "la imparcialidad no sólo es imposible, sino que el compromiso con este ideal tiene consecuencias ideológicas adversas" (Young, 2000, p. 190). Como especifica Palacio (2011), Young encuentra tres consecuencias adversas: la primera es que se hace cómplice del paradigma distributivo y sus males porque ambos requieren la neutralidad del Estado; la segunda, legitima los procesos burocráticos que impiden los democráticos porque requiere "una clase especial de ciudadanos que no estén implicados en la persecución de intereses privados en la sociedad de mercado, cuyo trabajo sea mantener el bien público y el punto de vista universal del Estado" (Young, 2000, p. 192); estos ciudadanos especiales son los burócratas; la tercera consecuencia adversa es que en lugar de eliminar la opresión termina reforzándola a través del imperialismo cultural, porque la perspectiva de los grupos dominantes terminaría siendo considerada la lógica imparcial a seguir.

Por lo tanto, para Young la injusticia no puede ser resuelta a partir de la imparcialidad. Por el contrario, Young encuentra en las 'políticas de la diferencia ${ }^{4 \text { ' }}$ la capacidad emancipadora necesaria para superar las injusticias. En lugar de plantear un Estado neutral, la apuesta de Young es un Estado inclusivo con los grupos sociales no dominantes que no exija la asimilación, sino que opere en la heterogeneidad. Por tanto, las personas no tienen que aceptar que su visión de la vida es menos valiosa que las dominantes para poder participar en los espacios de decisión política. La diferencia tendría un poder desestabilizador de las visiones de la vida dominante. Al afirmar el valor de los grupos sociales no dominantes la visión dominante deja de ser hegemónica y se convierte en una más que hace parte del juego político y debe dialogar con las otras. Pero, esta afirmación debe traducirse a acciones políticas concretas. Young sostiene que la afirmación requiere "cambios instituciones básicos [...] representación del grupo en la elaboración de políticas y la eliminación de la jerarquía de recompensa que fuerzan a las personas a competir por los escasos puestos más altos" (2000, p. 281). Lograr esto implica un marco de derechos básicos para todos, adicionado a un marco de derechos específicos para los grupos. En este sentido, la acción afirmativa que establezca un marco de derechos particular, siempre y cuando no reproduzca la visión menospreciada del grupo social, es la forma en la que según Young se elimina la opresión y la injusticia, incluso la económica.

\footnotetext{
${ }^{4}$ No todas las 'políticas de la diferencia' son consideradas por Young soluciones prometedoras a las injusticias. En el caso de las 'políticas de la diferencia' de tipo liberal, como las que propone Kymlicka en la Ciudadanía multicultural (1996) que consideran a los individuos antes que a los grupos sociales y terminan legitimando el imperialismo cultural. Véase Young (2009b) y Palacio (2011).
} 
En conclusión, Young diagnostica las injusticias en la medida en que los patrones institucionalizados limiten la participación de ciertos grupos en los espacios de toma de decisión, lo que implica también un proceso de dominación. Y la solución que sugiere para esta injusticia son las 'acciones afirmativas' que, siendo conscientes de la diferencia permanente de los grupos, diseña nuevos criterios para asignar puestos y posiciones sociales que permiten a los grupos visibilizarse y alcanzar participación en los espacios de toma de decisión.

\section{Nancy Fraser: Justicia en dos dimensiones}

En respuesta a la teoría de Young, Fraser construye un criterio normativo que procure la participación de todos eliminando los impedimentos culturales, que según Fraser son de los únicos que se ocupa Young, y de los económicos; este principio normativo es la 'paridad participativa' que consiste en la participación en pie de igualdad de todas las personas de una sociedad en los espacios de debate público para alcanzar consensos. Para hacerse efectiva, la 'paridad participativa' requiere dos condiciones previas de igualdad social que vayan más allá de la mera igualdad formal. Estas son las condiciones 'objetiva' y 'subjetiva 'que Fraser define así:

En primer lugar, la distribución de los recursos materiales debe hacerse de manera que garantice la independencia y la voz de todos los participantes. Llamo a esta condición objetiva previa de la paridad de participación [...] la segunda condición adicional para la paridad de participación la caracterizo como la condición intersubjetiva. Requiere que los patrones institucionalizados de interpretación y valoración expresen igual respeto por todos los participantes y aseguren igualdad de oportunidades para conseguir la estima social (1997, p. 42).

Las condiciones de la 'paridad participativa' marcan, además de un requisito, un veto a la agenda de discusión porque no se podría llegar a consensos que implique una pérdida de estas condiciones para algún participante. La paridad participativa exige una concepción bidimensional de la justicia que tenga en cuenta la redistribución y el reconocimiento (Cfr. Fraser, 1997). La redistribución satisface la 'condición objetiva', el reconocimiento la 'condición intersubjetiva'. Entonces, puede considerarse que la paridad participativa se hace efectiva cuando se supera la mala distribución y el mal reconocimiento ${ }^{5}$. En consecuencia, Fraser se enfrenta con las posiciones monistas de la justicia según las cuales la redistribución se recoge enteramente en el reconocimiento o viceversa. La autora nota que:

\footnotetext{
${ }^{5}$ Una brillante exposición de la evolución del concepto de 'paridad participativa' se hace en el artículo "La paridad participativa en la obra de Nancy Fraser" (2010) de la profesora Martha Palacio. En dicho artículo, la profesora Palacio ubica la definición de 'paridad participativa' como superación de la mala distribución y el mal reconocimiento en el momento que corresponde al modelo bidimensional y el libro Iustitia interrupta.
} 
Las exigencias de reconocimiento asumen a menudo la forma de un llamado de atención a la especificidad putativa de algún grupo, cuando no la crean efectivamente mediante su actuación y, luego, la afirmación de su valor. Por esta razón, tienden a promover la diferenciación de los grupos. Las exigencias de redistribución, por el contrario, abogan con frecuencia por la abolición de los arreglos económicos que sirven de soporte a la especificidad de los grupos (Fraser, 1997, pp. 25-26).

Siendo así las cosas, personas o grupos que tengan ambos tipos de reclamo, piden, por un lado, que su especificidad desaparezca mientras que por el otro que ésta se mantenga. Entonces icómo una concepción de la justicia puede hacer ambas cosas a la vez? Para responder esta pregunta debemos tener en cuenta la especificidad de cada dimensión de la justicia para ver cómo pueden unirse en una sola concepción y también la descripción que Fraser hace de los grupos lo que se corresponde con las dimensiones de la justicia.

Los reclamos de reconocimiento válidos, según Fraser, son aquellos que fomenten la 'paridad' y evitan crear nuevas desigualdades. Por tanto, las políticas del reconocimiento deberían propender por una igualdad formal sumada a una igualdad real, que se realiza con las condiciones de la paridad participativa para hacer que los grupos alcancen el estatus de par en la participación social a la vez que mantienen sus diferencias.

La injusticia derivada de la economía también debe resolverse, incluso antes que la que se deriva de los patrones culturales. Como bien dice Fraser la "imagen utópica de una cultura en la que construcciones de identidad y diferencia siempre nuevas sean elaboradas libremente y, luego, rápidamente deconstruidas es posible, después de todo, sólo sobre la base de una igualdad social básica" (1997, p. 49). Aquí, 'igualdad social básica' se refiere a unas condiciones materiales mínimas congruentes con una social democracia fuerte, que permitan una vida digna y a la vez permitan la lucha por el reconocimiento. El mensaje de Fraser es claro, antes de superar las injusticias culturales se deben superar las económicas.

La injusticia económica se presenta por lo general en forma de "explotación, marginación económica y ausencia de bienes materiales necesarios para llevar una vida digna" (Fraser, 1997, p. 21). Por tanto, el objetivo de una política redistributiva seria proveer a cada integrante de la sociedad de los elementos materiales y las condiciones laborales que le permitieran evitar alguno de estos tres problemas.

La redistribución es central para Fraser, sin embargo, no ofrece una teoría redistributiva específica, más bien supone que existe una injusticia económica que debe ser remediada por los medios que sean pertinentes. En palabras de Fraser, "no es necesario comprometerse con alguna explicación teórica en particular. 
Basta con suscribir una comprensión general de la injusticia socioeconómica, moldeada por un compromiso con el igualitarismo" (1997, p. 21). Lo único que debe considerarse, según Fraser, como solución para este tipo de injusticia "es algún tipo de reestructuración político-social. Esto puede implicar la redistribución del ingreso, la reorganización de la división del trabajo, el someter la inversión a decisiones democráticamente adoptadas, o la transformación de otras estructuras económicas básicas" (p. 24).

De esta forma, se empieza a reconciliar el paradigma de la redistribución con el paradigma de reconocimiento. En otras palabras, se supera el 'dilema redistribución-reconocimiento'. Esto es, si las demandas de reconocimiento de los grupos y sus características son abolidas por las demandas que piden igualdad de participación y estas, de carácter igualitario, se articulan con mayor facilidad con aquellas que piden condiciones de igualdad económica.

De acuerdo con esta versión los grupos que sufren injusticia económica son todos aquellos que se diferencian por el lugar que ocupan en una estructura económica injusta. También, que de darse únicamente justicia económica habrá más justicia que si existe únicamente justicia cultural. Esto porque, cómo se dijo al principio de la sección, la justicia económica se hace condición previa para obtener la justicia cultural. Además, que existen grupos que podrían asociarse con mayor facilidad a la dimensión cultural pero que tienen reclamos redistributivos. Como la injusticia está arraigada en la estructura económica, para superase, se debe alterar las estructuras económicas, estableciendo un sistema equilibrado de cargas y beneficios que elimine el modo de diferenciación. La consecuencia de esto sería la desaparición de la clase en cuestión y con ello la desaparición de la injusticia (Fraser, 1997).

Los grupos que padecen injusticia cultural-valorativa son aquellos que se distinguen por la forma en la que sus características son interpretadas y valoradas por los otros. De modo que su estatus de pares se vea limitado por 'patrones institucionalizados de valor'. El ejemplo del que se vale Fraser para explicar este tipo de grupos es el de las 'sexualidades menospreciadas'. Fraser afirma que:

La sexualidad es un modo de diferenciación social, cuyas raíces no se encuentran en la economía política, puesto que los homosexuales están distribuidos en toda la estructura de clases de la sociedad capitalista, no ocupan una posición especial en la división del trabajo y no constituyen una clase explotada (1997, p. 29) ${ }^{6}$.

\footnotetext{
${ }^{6}$ Aunque es muy probable que en el caso de la lucha homosexual real, se evidencie alguna limitación económica o subordinación laboral, para efectos de éste análisis no tendremos en cuenta la relación de las 'sexualidades menospreciadas' con los grupos homosexuales realmente existente.
} 
Las sexualidades menospreciadas (LGTB) sufren una injusticia que tiene un origen exclusivamente cultural y se da en función de la valoración que otros grupos tienen sobre estos y no sobre su posición económica o capacidad financiera. En este sentido y contrario a las teorías del reconocimiento que proponen el reconocimiento como una afirmación positiva de la particularidad grupal para evitar daño psicológico a quienes padecen el mal reconocimiento, Fraser enfoca su propuesta en la superación de los impedimentos culturales que le impiden a ciertos grupos participar en la esfera pública.

Fraser además, sugiere que existen grupos que sufren dos tipos de injusticia: económica y cultural. Estos grupos son los de 'género' y 'raza'. Las mujeres por ejemplo sufren injusticia económica por cuenta de la división del trabajo entre trabajos productivos y domésticos. Las mujeres que participan en los primeros se encuentran en muchas ocasiones sometidas a otra división desventajosa entre los trabajos de salarios altos y los de salarios bajos (Fraser, 1997). Asimismo, estas también se encuentran subordinadas culturalmente por el 'androcentrismo' que las ubica en un estatus bajo frente a los hombres.

Al enfrentar injusticia económica y cultural, los grupos bivalentes por lo general piden que sea abolida su diferenciación económica a la vez que luchan por que sea valorada su diferenciación cultural. En otras palabras "[...] los grupos bivalentes reclaman, por una parte, la redistribución de bienes teniendo como objetivo la disolución del grupo que sufre la injusticia y, por otro lado, al demandar el reconocimiento de sus diferencias, buscan fortalecer su identidad de grupo" (Fraser, 1997, p. 14).

Para solucionar en la práctica los problemas de los grupos, Fraser propone las 'acciones transformativas'. Definidas como "soluciones dirigidas a corregir los resultados inequitativos, precisamente mediante la reestructuración del marco general implícito que los origina. El punto esencial del contraste es resultados finales versus procesos que los generan, no el cambio gradual versus el apocalíptico" (Fraser, 1997, p. 32). Esta propuesta de solución, busca como fin último permitir las condiciones para la paridad participativa eliminando los obstáculos que impiden la participación.

\section{Debate entre Young y Fraser: ¿opresión o bidimensionalidad?}

Opresión y bidimensionalidad no parecen categorías intercambiables; opresión evoca algún tipo de totalitarismo político y la bidimensionalidad pareciera estar cerca de un método de investigación dual. No obstante, en la acepción de los términos que se usa aquí, tanto bidimensionalidad como opresión refieren a un entendimiento de la justicia y la injusticia. Estos términos son el foco del debate 
sostenido por Fraser y Young en la década de los noventa que se presentara a continuación.

La crítica de Fraser a Young tiene como punto central la aparente existencia de una bidimensionalidad de la justicia que es ignorada por Young. Fraser desestima la crítica de Young al paradigma distributivo valiéndose de la siguiente descripción:

Por un lado, [Young] recapitula la objeción marxista a las aproximaciones que se centran exclusivamente en patrones de resultados en lo relativo a la distribución de los bienes tangibles y las posiciones, tales como el ingreso y los empleos o cargos, pero deja de lado los procesos estructurales subyacentes que los producen. Aquí el blanco de la crítica es "el punto de vista de la distribución", por oposición al "punto de vista de la producción". Por otro lado, sin embargo, Young recapitula la objeción de Amartya Sen a las aproximaciones que se centran en la distribución de los bienes, por oposición a las capacidades, presentando a las personas como consumidores pasivos en lugar de agentes Aquí la crítica no está dirigida contra la distribución per se, sino contra la distribución del tipo equivocado de bienes. En un tercer aspecto, por último, la crítica de Young se dirige precisamente contra aproximaciones, como la de Sen, que tratan a lo intangible, como las capacidades, como centro de atención y objeto de la distribución. Aquí el blanco es la "reificación" (Fraser, 1997, pp. 253-254).

Los tres aspectos mantienen a Young en el marco del paradigma distributivo, según Fraser, porque recoge críticas que son propias de las teorías distributivas y sugieren salidas distributivas. Por ejemplo, la crítica de Young a las limitaciones de las capacidades de los grupos sociales como un efecto adverso del paradigma distributivo se inspira en la crítica de Amartya Sen a Rawls (1995), y su idea de 'bienes primarios' (1995), que lejos de alejarse del paradigma distributivo presenta elementos para fortalecerlo. El problema de Young, parece ser que limita demasiado los alcances de la distribución porque sus "juicios no implican necesariamente que las soluciones para la injusticia deban limitarse a medidas tales como la equiparación del ingreso a través de tributación redistributiva" (Fraser, 1997, p. 254). Por lo tanto, Young ignoraría las posibilidades de transformación que ofrecen las teorías distributivas.

Aunque Young se esfuerza por construir una crítica al paradigma distributivo para centrar la discusión en otros enfoques de la justicia, la distribución, según Fraser, aparece a lo largo de su obra como una preocupación implícita pero central. Por lo tanto, en la perspectiva de Fraser, Young construye el libro Justicia y la política de la diferencia (2000) usando como base un enfoque bifocal de la justicia, por un lado la redistribución y por el otro el reconocimiento. 
No obstante, Fraser concluye que Young ha culturizado en demasía su propuesta teórica debido al constante esfuerzo de Young por privilegiar la cultura sobre la economía. Esto se puede apreciar cuando Young sugiere que la justicia debe extenderse a una forma más general como la cultura y procede con este criterio a lo largo de su libro. Fraser intenta mostrar que, aunque Young haga uso de la cultura para explicar algunos conceptos clave como las cinco caras de la opresión y el concepto de justicia social, su modelo no deja de ser bidimensional.

Fraser categoriza las caras de la opresión de Young entre la cultura y la economía. La explotación, marginación y la carencia de poder son situadas por Fraser en la económica, porque estas caras de la opresión tienen su origen en procesos económicos, laborales y distributivos, que aunque son interpretadas por Young desde una perspectiva no distributiva más cercana a las ideas de Foucault sobre el poder, no dejan de ser una expresión de la injusticia económica.

El imperialismo cultural y la violencia se sitúan, según Fraser, en el espectro de la cultural. Estas últimas dos tienen como solución "[la revolución cultural]. Esto significa, fragmentar la idea de un solo conjunto universal de normas culturales y afirmar el pluralismo cultural y la diferencia" (Fraser, 1997, p. 265). Por su parte las caras de la opresión que se originan en la económica requieren "la reestructuración radical de la división del trabajo. Esto incluye eliminar la división, por ejemplo, entre el trabajo de definición de tareas y el de ejecución de las mismas, y ofrecer a todos actividades socialmente valoradas que desarrollen sus habilidades" (265).

Las características de estas dos dimensiones tienen, en principio, un carácter cultural. Sin embargo, Fraser encuentra en ellas componentes económicos debido a una cierta ambigüedad que pareciera subyacer a estas. En el imperialismo cultural, Young se inquieta por la universalización de una cultura pero parece ignorar, en la perspectiva de Fraser, que una cultura puede universalizarse a través de procesos político-económicos como en ciertos tipos de colonialismo. La violencia parece tener la misma situación porque puede surgir a partir de procesos culturales, pero también, en procesos económicos como las agresiones violentas por parte de representantes de las mayorías dominantes contra los inmigrantes en países desarrollados.

El problema que Fraser encuentra en este carácter bidimensional de las categorías de Young es que no logran reconciliar las soluciones a la injusticia cultural y económica. Por un lado, las caras de la opresión pueden requerir como solución, la política del reconocimiento que afirma las diferencias de grupo. Por otro lado, estas mismas pueden requerir soluciones económicas ligadas a la redistribución que tienden a eliminar la diferencia de grupo. Por lo tanto, la teoría de Young, aunque lograría hacer un diagnóstico de las injusticias no sería capaz de ofrecer una perspectiva viable de solución. 
Con esto Fraser pretende demostrar que cuando el origen de la injusticia no es cultural sino económico, la política de la diferencia carece de pertinencia e incluso puede jugar en contra de los intereses del grupo. En este tipo de injusticia, la justicia consiste en la eliminación del grupo que surge por la afinidad que una injusticia genera pero la política de la diferencia por el contrario, afirmaría la existencia del grupo lo que reforzaría la injusticia.

Young responde a Fraser criticando la distinción bidimensional y tratando de mostrar que seguir una concepción tal reforzaría algunas injusticias. La crítica de Young a Fraser, inicia con tres preguntas, que según ella, Fraser da por sentado en la condición 'postsocialista':

¿Olvidan la economía política los teóricos de la justicia? ¿Buscamos raíces culturales en los determinantes de las injusticias más importantes? ¿Ya es el momento de la teoría social crítica para reafirmar una distinción básica entre procesos materiales de la economía política y los procesos simbólicos de la cultura? (Young, 2009, p. 193) ${ }^{7}$.

Ya que Fraser responde afirmativamente a estas preguntas entonces se preocupa por proveer una teorización que incluya la redistribución al lado del reconocimiento para evitar el olvido de la economía política, a la vez que busca las raíces materiales de las injusticias para evitar que se expliquen únicamente desde la cultura y distingue de forma analítica los procesos materiales y simbólicos. Contrario a Fraser, Young respondería negativamente estas preguntas, diría que los teóricos de la justicia no han olvidado la economía política porque se encuentra implícita en los procesos culturales y que no hace falta una distinción radical entre los procesos materiales y culturales. A partir de esta posición Young enfila su batería crítica hacia Fraser.

La crítica central es que una concepción de la justicia social con dos dimensiones diferentes y excluyentes es una estrategia polarizadora y no permite analizar las injusticias de forma adecuada. Young entiende que Fraser construye un artificio analítico donde redistribución y reconocimiento son diametralmente opuestas. A partir de esto, Young reconoce que: "La distinción entre redistribución y reconocimiento es [...] una distinción analítica necesaria para la construcción de su exposición" (Young, 2009, p. 196) ${ }^{8}$. Pero, encuentra un problema adicional, el modelo dicotómico de redistribución-reconocimiento

\footnotetext{
${ }^{7}$ La traducción es mía. El texto original reza así; “Esqueceram-se da economia política, os teóricos da Justiça? Buscamos em raízes culturais os determinantes das injustiças mais importantes? Já é tempo de a teoria social crítica reafirmar uma distinção básica entre os processos materiais da economia política e os processos simbólicos da cultura?"

${ }^{8}$ La traducción es mía. El texto original reza así: "A distinção entre redistribuição e reconhecimento é [...] uma distinção analítica necessária para a construção da exposição".
} 
parece no tener espacio para elementos adicionales que se quedan por fuera de la dicotomía, específicamente el aspecto político. En el ensayo de Fraser, al que Young refiere, se había argumentado a favor de mantener la dimensión política unida a la economía cobijados por la redistribución. En esto, Fraser sigue a Habermas afirmando que: "Mi inclinación es seguir a Jürgen Habermas, viendo estas cuestiones de forma bifocal. De una perspectiva, las instituciones políticas (en las sociedades capitalistas con regulación estatal) pertenecen a la economía, como parte del "sistema" que prod uce injusticias distributivas socio-economicas" (Fraser, 1995. Citada en Young, 2009, p. 199) ${ }^{9}$. Con esta unión entre economía y política, Fraser deja por fuera la posibilidad de un paradigma de justicia de representación ${ }^{10}$.

Contraria a Fraser, Young cree que el reconocimiento tiene influencia en asuntos de carácter redistributivo. En el caso de las sexualidades menospreciadas, que Fraser usa para ejemplificar la injusticia cultural, la perspectiva de Young (2009) apunta al heterosexismo como causante de injusticia cultural y económica. Las personas pertenecientes a la comunidad LGTBI son menospreciados culturalmente pero también se encuentran limitados para acceder a la protección social, salud, pensiones, adopción, etc. ${ }^{11}$. Estas desventajas, se encuentran ligadas a lo económico, pero se resolverían desde la cultura atacando el heterosexismo. Para esta relación no iría en una sola dirección, la economía también influiría en la cultura.

\section{El balance ¿Fraser o Young?}

La pregunta a abordar ahora es la siguiente ¿cuál autora ofrece una mejor interpretación de la injusticia y el remedio necesario? Para responder analizaré por separados los argumentos de cada autora para mostrar sus fortalezas y debilidades y para hacer el balance y determinar cuál teoría es más prometedora.

El modelo que produce Young tiene tres virtudes: la primera y quizá más importante es su crítica al paradigma distributivo; la segunda consiste en la relación

\footnotetext{
9 Traducción propia. El texto original reza así; Minha inclinação é acompanhar Jürgen Habermas, vendo tais questões de maneira bifocal. De uma perspectiva, as instituições políticas (nas sociedades capitalistas com regulação estatal) pertencem à economia, como parte do "sistema" que produz injustiças distributivas sócio-econômicas.

${ }^{10}$ Fraser corrige este olvido en Escalas de justicia (2008), sin embargo, en el momento en que Young formula la crítica Fraser no había conceptualizado lo político como algo independiente de la economía. Siendo así las cosas, las categorías analíticas de Fraser, redistribución y reconocimiento, se hacen rígidas e inamovibles.

${ }^{11}$ En el momento en el que Young presenta éste ejemplo, los derechos de la comunidad LGBT estaban muy restringidos, dependiendo del país, hoy esta comunidad goza de un paquete de derechos más amplio, lo que desactualiza la lista de desventajas que Young (2009) enuncia.
} 
que traza entre la imparcialidad y la opresión; la tercera es su contribución a la discusión por la diferencia y la diversidad en las sociedades contemporáneas.

El aporte más importante de Young ha sido su crítica al paradigma distributivo. Este paradigma que existe desde Aristóteles, y sus reflexiones sobre la justicia, efectivamente esconde el marco institucional o los modos de producción, en sentido marxista, en los que se da la distribución. Por tanto, centrar la justicia exclusivamente en este paradigma tiende a ignorar injusticias asociadas a ese marco ignorado. En esto, Young tiene una gran virtud.

Sumado al ocultamiento del contexto, el paradigma distributivo se vale de la idea de imparcialidad para justificar la distribución de los bienes. Por tanto, olvida las diferencias y tiende a construir una sola subjetividad que opera al nivel de la política excluyendo las diferencias de los espacios de toma de decisión; además, excluye el contenido moral de los grupos del debate público para mantener la idea de imparcialidad. Mostrar esta conexión y la limitación que la justicia centrada en la distribución puede ejercer en la capacidad de participación política de los grupos excluidos.

También, el aporte de Young frente a la diferencia y la diversidad se hace central en las discusiones posteriores sobre la justicia, especialmente las teorizaciones feministas; muestra de ello es Fraser quien funda gran parte de su teoría bidimensional en una crítica a Young. La construcción de su definición de grupo asociada a los modos de vida y la necesidad de afirmar la diferencia de los grupos para remediar la injusticia es una virtud de Young.

Sin embargo, la vehemencia de Young en su crítica al ideal de imparcialidad puede tener una segunda cara no tan positiva. Mi evaluación de las debilidades de Young parte de la amplitud con la que Young entiende la imparcialidad y que tiene efectos en la propuesta de política de la diferencia Además, crítico que Young intente reinterpretar los problemas económicos desde una perspectiva cultural cuando su propuesta inicial no es eliminar el paradigma distributivo sino desplazar su centralidad en la discusión. Procedo a presentar con mayor claridad estas dos debilidades.

La crítica de Young contra la imparcialidad se ha dirigido a la tradición distributiva que efectivamente ha obviado o ignorado la diversidad de concepciones de vida buena; muestra de ese olvido es el giro que Rawls da en el Liberalismo Político (2006) respecto de su primera versión, incluyendo la diversidad de 'doctrinas comprehensivas de lo bueno' en el concepto de 'razón publica'. Sin embargo, Young no limita su crítica a la imparcialidad y parece extenderla a cualquier presentación limitando su función a tres aspectos. 


\begin{abstract}
Sustenta la idea del estado neutral, que a su vez sirve en parte de fundamento al paradigma distributivo de justicia. Legitima la autoridad burocrática, asî como los procesos jerárquicos de toma de decisiones democráticamente tomadas. Y por último, refuerza la opresión al transformar el punto de vista de los grupos privilegiados en una posición universal. Sostengo que, en lugar de la imparcialidad deberíamos buscar la equidad social en un contexto de heterogeneidad y discurso parcial (Young, 2000, p. 190).
\end{abstract}

Aquí, podemos evidenciar que Young limita los efectos de la imparcialidad al refuerzo de la injusticia. El problema de universalización de la crítica de Young al ideal de imparcialidad radica en que niega también la utilidad de la imparcialidad en la ética comunicativa ${ }^{12}$ que ella misma acepta como necesaria ${ }^{13}$. El profesor Ivan Teimil en su artículo "Justicia como imparcialidad dialógica. Una perspectiva de la justicia imparcial compatible con las demandas de los grupos desfavorecidos" (2012) propone que Young, al no considerar la necesidad de la imparcialidad en la ética comunicativa, contradice su propuesta de justicia social anclada en la política.

El argumento del profesor Teimil sugiere que para hacer coherente la propuesta de Young con la ética comunicativa, se debe aceptar la imparcialidad deliberativa-dialógica que consiste en "En expresión común, la imparcialidad se refiere a «calzarse los zapatos del otro» sin eludir la responsabilidad de admitir las reclamaciones de quienes padecen desventajas y siendo igualmente conscientes de los límites democráticos a tales reclamaciones" (Teimil, 2012, p. 593). En este sentido, se requiere de una imparcialidad dialógica que sustente el proceso democrático de toma de decisiones que Young pone como condición necesaria de la justicia social.

Debido al rechazo a la imparcialidad, Young discute las teorías distributivas que por lo general articulan el ideal de imparcialidad para determinar la justa distribución de cargas y beneficios. Según mi análisis, la debilidad mostrada termina derivando en su segunda debilidad: Young no logra explicar con sus categorías las injusticias económicas ni propone una solución a las injusticias de este tipo. Es justo decir que la injusticia económica no es el problema central del modelo de Young, sin embargo, las integra en su obra desde la crítica y algunas caras de

\footnotetext{
${ }_{12}$ Habermas en Verdad y Justificación (2002) suscribe la idea de imparcialidad en sentido dialógico para legitimar los acuerdos alcanzados en la esfera pública. Dice puntualmente que "La expectativa de legitimidad —según la cual sólo pueden merecer reconocimiento aquellas normas que «son buenas para todos en la misma medida»— se puede satisfacer hoy solamente con la ayuda de un procedimiento que, bajo condiciones de inclusión de todos los potencialmente afectados, asegure la imparcialidad en el sentido de atender en la misma medida todos los intereses implicados" (Habermas, 2002, p. 292).

${ }^{13}$ Young sugiere que la democracia y la participación política son condiciones necesarias para alcanzar la justicia social. Recurre a Habermas y la ética comunicativa para apoyar su tesis. Esto puede ampliarse con una lectura de (Young, 2000, pp. 159-161).
} 
la opresión, donde a través de una reinterpretación de la injusticia económica, intenta darle una nueva perspectiva en clave cultural y de capacidad.

La versión de Fraser que se ha visto hasta el momento tiene dos grandes virtudes: reconcilia el paradigma distributivo con las políticas del reconocimiento, clasifica los remedios a las injusticias de modo que permite diferenciar las formas de enfrentar la injusticia de acuerdo con su pertinencia. Sin embargo, aún no logra superar el carácter analítico de la justicia bidimensional lo que le imposibilita hablar con pericia de las injusticias reales porque no logra explicarlas completamente. Explico a continuación con más detalle.

La época que Fraser llama 'postsocialista' está marcada por el desplazamiento de las luchas por la igualdad social que cayeron en descredito después de la caída del socialismo realmente existente. Además, casi al mismo tiempo, emerge de la mano de Taylor (1997), Honneth (1997) y en cierta medida Young (2000), una nueva forma de interpretar las luchas sociales como luchas por el reconocimiento de la diferencia.

Fraser entiende que esa nueva 'gramática de las luchas sociales' asociada al reconocimiento expresa parte de los reclamos de justicia de las víctimas. Sin embargo, considera que es equivocado eclipsar la lucha por la igualdad social, aun no resuelta, en la lógica del reconocimiento porque las injusticias económicas no eran interpretadas adecuadamente en esta lógica.

Siguiendo el proyecto trazado en el artículo "La lucha por las necesidades. Esbozo de una teoría socialista-feminista de la cultura política en el capitalismo tardío" (1991) Fraser propone 3 momentos para interpretar las luchas por las necesidades: el primero "es la lucha por establecer el estatuto político de una necesidad dada, la lucha por validar la necesidad como un asunto político" (Fraser, 1991, p. 3). Este paso ya habría sido dado por el reconocimiento y también por las luchas redistributivas. El segundo momento consiste en "la lucha por la interpretación de la necesidad" y el tercero en la implementación de las medidas que solucionan la necesidad.

Fraser considera que ambas necesidades, redistribución y reconocimiento, han establecido su estatus político, lo que se debe hacer es tramitar el segundo paso referente a la interpretación de la necesidad. Allí, es donde se sitúa el desarrollo teórico de Fraser al que me he referido en este trabajo, Fraser se encuentra en la lucha por interpretar estas necesidades y lo hace de forma independiente pero relacionada; considera que redistribución y reconocimiento parten de necesidades diferentes y por tanto, deben tener categorías, interpretaciones y soluciones independientes, sin embargo, deben reconciliarse para evitar que las soluciones de una y otra necesidad sean contrapuestas. 
La clasificación que Fraser hace de las soluciones o remedios a la injusticia, es una gran virtud de su obra porque resuelve el problema de la contraposición en la integración de la redistribución y el reconocimiento. Ciertamente, las políticas afirmativas culturales tienen como consecuencia el reforzamiento de las identidades de grupo igual que las acciones afirmativas redistributivas, la cuestión es que el reforzamiento de los grupos puede constituir nuevas injusticias ${ }^{14}$.

Por lo anterior, la inserción de las acciones transformativas permite una integración de las luchas por la justicia cultural y la justicia económica porque tanto la acción transformativa cultural como la económica tienen como objetivo la desaparición del grupo y eliminando así la injusticia. El lector podría pensar entonces que Fraser es una asimilacioncita que busca homogenizar a los grupos para superar la injusticia pero, teniendo en cuenta la distinción de las diferencias de grupo que Fraser propuso, la desaparición de grupo refiere a la diferencia que producida por la injusticia, no por las diferencias que deben universalizarse o disfrutarse; en este sentido, la diversidad se mantiene; así, Fraser intenta superar la crítica de Young según la cual redistribución y reconocimiento son categorías excluyentes.

Fraser sugiere que las injusticias se pueden dar por motivos económicos, culturales o una mezcla de ambos. La dificultad se presenta porque no considera los alcances de la bidimensionalidad de la injusticia lo que termina haciendo que su teoría se aleje de la realidad social. Aunque Fraser prevé la existencia de grupos bivalentes limita en demasía su alcance. Fraser, en su afán de hacer una consideración analítica y necesaria de la injusticia, olvida aterrizar esta distinción al plano práctico lo que implica la yuxtaposición de diversos tipos de injusticia en el mismo grupo social. En resumen, el problema de Fraser fue no considerar el alcance de la bidimensionalidad y teorizar con profundidad sobre esto en el nivel de teoría social.

No obstante, la posición de Fraser ofrece ciertas ventajas prácticas que no ofrece Young. Puntualmente tres aspectos: el primero consiste en la integración de redistribución y reconocimiento en una sola concepción de la justicia sumada a la definición de grupo subordinado que se encuentra en función de dicha integración. El segundo, radica en la función de la 'paridad participativa' como criterio de validación de las demandas de los grupos. El tercero y último radica en la clasificación de los remedios a las injusticias que diseña Fraser y se pone en función de la integración de economía y la cultura. De acuerdo a estas ventajas, considero que la propuesta de Fraser es más prometedora y, sin obviar las virtudes de Young, es la perspectiva en la que se debe avanzar.

\footnotetext{
${ }^{14}$ Esto aplica para los grupos que se constituyen a partir de diferencias asociadas a injusticias como las diferencias de clase uno en el modelo de 'teoría critica del reconocimiento' de Fraser que se expuso en el punto 2.1 de este texto.
} 
Para explicar el primer aspecto, se debe recordar que la opinión de Young es que las acciones afirmativas de carácter no redistributivo, aquellas que le dan valor positivo a los grupos sociales, socaban también la injusticia económica. Así, en la perspectiva de esta pensadora, al eliminar la opresión que sufren los grupos también se mejoran las condiciones materiales de sus integrantes. Esta postura está fundada en la idea de que las condiciones culturales determinan las económicas y viceversa, permitiendo a Young formular una crítica a las categorías redistribuciónreconocimiento de Fraser.

En lugar de apoyar la propuesta de Young, la idea de la reciprocidad entre cultura y economía, termina por demostrar que Fraser tiene una visión más amplia de la injusticia que la de Young, quien se ve impedida para explicar todas las injusticias económicas y termina por quedarse solo con aquellas que son culturales.

Supongamos que una fábrica se ve obligada a despedir a cincuenta empleados de rango operativo porque ha comprado una máquina que hace el trabajo de estos empleados; para determinar quiénes serán despedidos la empresa usa el criterio de productividad, los cincuenta empleados que tienen menor índice de productividad serán despedidos. Entre los 50 empleados se cuentan musulmanes, cristianos, negros, mujeres, homosexuales, profesionales y no profesionales. El origen del problema en este caso es económico, aunque algunos trabajadores se cuenten entre grupos sociales subordinados y pueda derivar en injusticia cultural. Young sugeriría en este caso que el problema de justicia es que los operarios al tener el estatus más bajo sufrieron opresión en términos de 'carencia de poder'. Hasta aquí tiene sentido la perspectiva de Young ya que fueron despedidos los que carecen de poder, quienes pueden ejercerlo siguen con sus trabajos. No obstante, la perspectiva de esta pensadora ignora que el origen de la injusticia no es la 'carencia de poder' sino el criterio de eficiencia económica que impulsa a los dueños de la empresa a prescindir de los empleados por una máquina que hace el mismo trabajo más rápido y en menos tiempo. Si se aplica el enfoque bidimensional de Fraser a esta misma situación, se detectará inmediatamente el origen económico de la injusticia, a la vez que se encontraría la injusticia cultural que se deriva de dicha injusticia económica.

El segundo aspecto es que Fraser ofrece un criterio para determinar cuáles demandas de justicia son válidas y cuáles no. Se debe tener en cuenta que tanto Fraser como Young están del lado de las víctimas, por eso intentan interpretar y ofrecer soluciones a la mayor cantidad posible de víctimas de injusticias. Ambas construyen sus argumentos desde el clamor de las víctimas. Young centra su análisis en el caso norteamericano mientras Fraser hace un intento de carácter universalista. Debido a esto, Young teoriza a partir de una experiencia concreta, sin verse en la necesidad de ofrecer un principio normativo ni construir una teoría de la justicia; como bien dice Young al inicio de su libro La justicia y la política de la diferencia (2000) "a pesar de que analizo la justicia y argumento sobre ella, 
no construyo una teoría de la justicia" (p. 12). Fraser por el contrario, intenta construir elementos propios de una teoría de la justicia que tiene como eje central la 'paridad participativa'.

Si se pasan las demandas de justicia por el filtro de estas pensadoras, se obtendrán resultados distintos. En el caso de Young y su énfasis en la diferencia, todas las demandas de justicia que provengan de grupos sociales construidos a partir de un sentido de identidad, al provenir de oprimidos deben ser remediadas con acciones afirmativas. De manera que Young, probablemente sin quererlo, termina legitimando todas las demandas de justicia sin aplicar un criterio que determine cuál es válida y cual no; como bien sugiere la profesora Avendaño (2013) "El énfasis en las diferencias, en el caso de Young, conduciría a que éstas fueran afirmadas de un modo implícito, sin que contemos con un criterio evaluativo para determinar con cuáles nos quedamos y cuáles desechamos" (p. 85). En el caso de Fraser, la 'paridad participativa' sirve como criterio de validación de las demandas de justicia. Como se mencionó al inicio, los grupos que promuevan la 'paridad de participación' y no fomenten la desigualdad son aquellos que pueden presentar reclamos válidos, cualquier grupo que promueva la inferioridad de otros no puede presentar un reclamo valido.

A la hora de diseñar políticas concretas que resuelvan las injusticias, una propuesta como la de Young da por sentado que la opresión y sus cinco caras marcan el camino a seguir en términos de soluciones políticas; en otras palabras, el tipo de opresión determina la solución adecuada. El problema de esta perspectiva es que a priori determina que la solución es de carácter cultural y los problemas económicos serán resueltos al ser una derivación de la cultura.

Por el contrario, en el enfoque bidimensional actúan a posteriori como elementos de análisis que sirven para determinar qué tan económica es la injusticia y cuál es su relación puntual con la cultural o, por el contrario, que tan cultural es la injusticia y cuál es su relación con la económica. La ventaja puntual es que, la propuesta de Fraser brinda herramientas de análisis de las injusticias particulares para determinar con claridad que es lo que se debe transformar en la sociedad y el Estado para solucionar la injusticia evitando la mayor cantidad posible de efectos adversos.

\section{Conclusiones}

Después de hacer un recorrido por las teorías de Fraser y Young y hacerlas dialogar para revisar las propuestas de diagnóstico y remedios a la injusticia, se puede concluir que: (i) La teoría de Fraser surge, en gran medida, como una respuesta a la de Young y se va permeando por esta a lo largo del debate y en sus desarrollos posteriores. (ii) Una perspectiva de la justicia que integre diferentes 
paradigmas normativos permite un diagnóstico más completo y emancipador que los intentos por reducir la interpretación de las injusticias en un solo paradigma normativo. (iii) Los remedios a la injusticia que eliminen las estructuras generadoras de la injusticia son más deseables que aquellas que no afectan dichas estructuras.

(i) En el libro lustitua interrupta (1997) Fraser expone la necesidad de integrar dos paradigmas normativos que se mostraban opuestos, la redistribución y el reconocimiento. Esa integración responde a la propuesta de Young de desplazar el paradigma distributivo por las injusticias que se derivan de este. Así, la propuesta de Young es una respuesta a las críticas de Young al paradigma distributivo. Asimismo, Fraser no solo discute ese punto, sino que discute con la política de la diferencia, la acción afirmativa y la clasificación de los grupos. En cada caso Fraser propone una salida opuesta a la de Young.

En este sentido, la propuesta de Fraser intenta ofrecer una teoría creíble que reivindique la redistribución sin desconocer las nuevas injusticias que se tematizaban desde el paradigma del reconocimiento. Aunque Young no abogaba directamente por el reconocimiento, al menos esa no era la palabra que ella empleaba, sí reinterpretaba la justicia desde la cultura, incluso aquellas cuestiones tradicionalmente asociadas a la redistribución.

(ii) Esto permite ver cómo dos propuestas similares que problematizan sobre los mismos asuntos, ofrecen soluciones diferentes, una fundada en la diferencia y otra en el igualitarismo. Parece entonces que esta última tiene ventajas sobre la primera porque propone un tipo de justicia social amplio que incluye la dimensión económica, la cultural y la política, pero que está abierto a nuevas tradiciones normativas y así evita la dificultad de reducir una dimensión en otra de forma satisfactoria a la vez que queda presta para evolucionar conforme cambien las injusticias. Así, Fraser supera la dificultad de los monismos que se ven es aprietos para explicar todo tipo de injusticias desde un solo paradigma normativo.

(iii) Como los grupos son las víctimas de la injusticia, debido a que las diferencias particulares de cada uno podían ponerlo en ventaja o desventaja, la tematización de la justicia se hizo, en las dos autoras, pensando en grupos sociales subordinados. Por tanto, los remedios a la injusticia debían afectar las diferencias que daban pie a la injusticia. Young proponía afirmar las diferencias de los grupos para empoderarlos y ampliar su capacidad de participación en las esferas públicas.

Las soluciones de Fraser se diferencian de la afirmación porque atacan el origen de las injusticias, su enraizamiento en el sistema económico y cultural. Por proponer cambios en estructuras tan arraigadas y fuertes, Fraser sacrifica gran parte de la factibilidad de su propuesta, pero atina al usar la afirmación como agente de cambios más pequeños que, sin embargo, pueden producir grandes cambios a largo plazo. Por ejemplo, un congreso integrado por grupos sociales 
y no por partidos políticos o grupos de conformación diversa, tendría debates constantes sobre la forma de estimular la diferencia y el modus vivendi de esos grupos, cosa que Fraser no vería como positiva. Una 'acción afirmativa' que se vea realizada en un modelo como el anterior difícilmente puede ser considerada una solución viable porque, en lugar de fomentar la convivencia y el reconocimiento mutuo, termina en una negociación eterna sobre las prebendas que cada grupo representado debe tener.

\section{Referencias}

Fraser, N. (1994) La lucha por las necesidades: Esbozo de una teoría critica socialista-feminista de la cultura política del capitalismo tardío. Revista Debate Feminista, (3), 3-40.

Fraser, N. (1997). Iustitia Interrupta. Bogotá: Siglo del Hombre Editores.

Habermas, J. (2002). Verdad y justificación. Madrid: Editorial Trotta.

Palacio, T. (2011). Justicia y diferencia en Iris Marion Young. Eikasia. Revista de Filosofía, V(39), 74-106.

Peréz, O. (2010). Escalas de justicia y emancipación: Inclusión, redistribución y reconocimiento. Astrolabio. Revista internacional de filosofía, 2010(11), 378-391.

Teimil, I. (2012). Justicia como imparcialidad dialógica. Una perspectiva de la justicia imparcial compatible con las demandas de los grupos desfavorecidos. Revista Isegoría, (47), 587-600.

Young, I. (2000). La justicia y la política de la diferencia. (S. Álvarez, Trad.). Madrid: Ediciones Cátedra.

Young, I. (2009). Categorías desajustadas: Uma crítica à teoria dual de sistemas de Nancy Fraser. Revista Brasileira de Ciência Política, (8), 193-214. 